\title{
Effects of mesonic correlations in the QCD phase transition
}

\author{
D. Blaschke,,$^{1,2, *} \quad$ M. Buballa, ${ }^{3, * *}$ A. E. Radzhabov, ${ }^{2, * * *}$ and M. K. Volkov ${ }^{2, * * *}$ \\ ${ }^{1}$ Institute for Theoretical Physics, University of Wroclaw, 50-204 Wroclaw, Poland \\ ${ }^{2}$ Bogoliubov Laboratory of Theoretical Physics, JINR Dubna, 141980 Dubna, Russia \\ ${ }^{3}$ Institut für Kernphysik, Technische Universität Darmstadt, D-64289 Darmstadt, Germany
}

The finite temperature phase transition of strongly interacting matter is studied within a nonlocal chiral quark model of the NJL type coupled to a Polyakov loop. In contrast to previous investigations which were restricted to the mean-field approximation, mesonic correlations are included by evaluating the quark-antiquark ring sum. For physical pion masses, we find that the pions dominate the pressure below the phase transition, whereas above $T_{c}$ the pressure is well described by the meanfield approximation result. For large pion masses, as realized in lattice simulations, the meson effects are suppressed.

The interest in the phase diagram of quantum chromodynamics (QCD) has received new impulses from the recent results of heavy-ion collision experiments at RHIC Brookhaven [1], revealing that hot hadronic matter at temperatures not too far above the critical temperature $T_{c}$ behaves like a perfect liquid, rather than a weakly coupled plasma. A theoretical description of these investigations therefore requires a non-perturbative approach, which also provides a proper understanding of the chiral quark dynamics and the confinement mechanism. Until now, the only method which is directly based on QCD and which meets these requirements is lattice gauge theory. Unfortunately, the application of lattice results to experimental data is complicated by the fact that most lattice calculations are performed with rather large quark masses, leading to unphysically large pion masses. Therefore, in spite of recent progress in this aspect, additional methods are required to extrapolate the lattice results to the physical masses. To bridge the gap there are major efforts within chiral perturbation theory $(\chi P T)$ (see, e.g., [2]). However, $\chi P T$ is obviously not suited to describe

\footnotetext{
* Electronic address: blaschke@ift.uni.wroc.pl

** Electronic address: michael.buballa@physik.tu-darmstadt.de

*** Electronic address: aradzh@theor.jinr.ru

${ }^{* * * *}$ Electronic address: volkov@theor.jinr.ru
} 
the region of the phase boundary or even beyond. In this regime, one has to employ other approaches for the chiral extrapolation, like QCD Dyson-Schwinger equations (see, e.g., [3]) or effective models which share the relevant degrees of freedom with nonperturbative QCD [4]. These approaches have the additional advantage that they can straightforwardly be extended to nonzero chemical potential, which is still a serious problem on the lattice.

In this article we want to discuss an effective model of low-energy QCD, capable of describing the chiral as well as the deconfinement transitions. As a basis we use the PNJL model [5-8] which generalizes the well-known Nambu-Jona-Lasinio (NJL) model [9] for the chiral quark dynamics by coupling it to the Polyakov loop, which serves as an order parameter of the deconfinement transition. To a large extent, this removes one of the most disturbing features of the original NJL model, namely the pressure contribution of unconfined quarks in the hadronic phase. In spite of the simplicity of the model a remarkable agreement with the results of lattice QCD thermodynamics [10, 11] has been obtained [5].

However, this comparison was not entirely consistent: Whereas unphysically large values of the current quark masses have been used in the lattice simulations [10, 11], physical values have been employed in the PNJL model analysis of Ref. [5]. Moreover, after successfully removing (most of) the unphysical quark degrees of freedom from the confined phase, the PNJL model treated in mean-field approximation as in Ref. [5] does not contain any degree of freedom in this regime. Obviously, this is a rather poor description of the hadronic phase at finite temperature where mesons are expected to become relevant. Hence, the good agreement of the PNJL results with the lattice data could partially be accidental in that mesonic correlations have been neglected in the PNJL analysis, while in the lattice calculations they are suppressed by the large current quark masses. To get a consistent picture it is thus important to go beyond the mean-field approximation and to include mesonic correlations. This can be done systematically within the framework of a $1 / N_{c}$ expansion scheme [12], which has successfully been applied to the NJL model, e.g., in Refs. [13].

In the present work we suggest a $1 / N_{c}$ improvement of the PNJL model which is necessary to disentangle hadronic contributions in the vicinity of and below the chiral/deconfinement phase transition. Moreover, the existence of bound states above $T_{c}$ may be crucial for the understanding of the properties of the strongly coupled quark-gluon plasma [14]. A consistent inclusion of hadron gas contributions should thus include the dissociation of hadrons being bound states of quarks and antiquarks below the transition into resonant continuum 
correlations somewhere above it. This hadronic Mott-transition [15] has been discussed first in Refs. [16] and was formulated within the NJL model in Ref. [17] employing the concept of spectral functions and in-medium scattering phase shifts within a Beth-Uhlenbeck approach, see [18].

In this letter, we restrict ourselves to the case of zero chemical potential. The central quantity for our analysis is then the thermodynamic potential at finite temperature which can be written as a sum of a mean-field part and a part which describes the mesonic correlations,

$$
\Omega(T)=\Omega_{\mathrm{mf}}(T)+\Omega_{\mathrm{corr}}(T)-\Omega_{0}
$$

As usual, we have introduced an irrelevant constant $\Omega_{0}$, which is chosen such that $\Omega(0)=0$. Technically, the correlation part $\Omega_{\text {corr }}(T)$ corresponds to a ring-sum of quark-antiquark loops (see $[17,19])$. For the evaluation of $(1)$, we consider a nonlocal generalization of the PNJL model. The nonlocal four-point interaction is chosen in a separable form motivated by the instanton liquid model (ILM) [20] where it results from the internal nonlocal structure of the nonperturbative QCD vacuum. In the ILM, the nonlocality is represented by the profile function of the quark zero-mode in the instanton field and depends on the gauge. We use a Gaussian ansatz as one of the simplest functional forms of the nonlocality which has a similar behavior as the zero-mode profile obtained in a gauge invariant manner [21]. This choice guarantees convergence at all orders without an additional regularization procedure.

The quark sector of the nonlocal chiral quark model is described by the Lagrangian

$$
\mathcal{L}_{q}=\bar{q}(x)\left(i \not D-m_{c}\right) q(x)+\frac{G}{2}\left[J_{\sigma}^{2}(x)+\vec{J}_{\pi}^{2}(x)\right]
$$

where $m_{c}$ is the current quark mass, and $D_{\mu}=\partial_{\mu}-i A_{\mu}$ the covariant derivative with a background gluon field $A_{\mu} \equiv A_{\mu}^{a} \frac{\lambda^{a}}{2}=\delta_{\mu 0} A_{0}$. The nonlocal quark currents are

$$
J_{I}(x)=\int d^{4} x_{1} d^{4} x_{2} f\left(x_{1}\right) f\left(x_{2}\right) \bar{q}\left(x-x_{1}\right) \Gamma_{I} q\left(x+x_{2}\right),
$$

with the vertices $\Gamma_{\sigma}=\mathbf{1}$ and $\Gamma_{\pi}^{a}=i \gamma^{5} \tau^{a}, a=1,2,3$.

After linearization of the four-fermion vertices by introducing auxiliary scalar $(\tilde{\sigma})$ and pseudoscalar $\left(\pi^{a}\right)$ meson fields the quark sector is described by the Lagrangian

$$
\mathcal{L}_{q \pi \sigma}=\bar{q}(x)\left(i \not D-m_{c}\right) q(x)-\frac{\pi_{a}^{2}+\tilde{\sigma}^{2}}{2 G}+J_{\sigma}(x) \tilde{\sigma}(x)+\pi^{a}(x) J_{\pi}^{a}(x) .
$$

To proceed, we single out the nonzero mean-field value of the scalar field by the decomposition $\tilde{\sigma}=\sigma+\sigma_{\mathrm{mf}}$ so that $\pi^{a}$ and $\sigma$ denote only the fluctuating parts of the fields $\left(\left\langle\pi^{a}\right\rangle=\langle\sigma\rangle=0\right)$ 
describing mesonic correlations. The scalar mean field gives a dynamical contribution to the quark mass which in Euclidean momentum space is $M\left(p^{2}\right)=m_{c}+m_{d} f^{2}\left(p^{2}\right)$ with $f^{2}\left(p^{2}\right)=$ $\exp \left(-p^{2} / \Lambda^{2}\right)$ being the (Fourier transformed) Gaussian form factor in Euclidean space. The amplitude $m_{d}=-\sigma_{\mathrm{mf}}$ is an order parameter for dynamical chiral symmetry breaking. The model parameters of the quark sector are fixed by using the pion mass and the weak pion decay constant and choosing a chiral condensate value in accordance with the limits from QCD sum rule analyses. The chiral condensate is obtained from the non-perturbative part of the quark propagator, $S_{n p}(p)=\left(\not p+M\left(p^{2}\right)\right)^{-1}-\left(\not p+m_{c}\right)^{-1}$, i.e., after subtracting the perturbative part. For details see, e.g., [22, 23]. For the analyses in the present paper, we employ a parameter set from Ref. [22] for $-\langle\bar{q} q\rangle^{1 / 3}=240 \mathrm{MeV}$ given by $m_{c}=5.8 \mathrm{MeV}$, $\Lambda=902.4 \mathrm{MeV}$ and $G \Lambda^{2}=15.82$.

The mean-field thermodynamic potential reads

$$
\Omega_{\mathrm{mf}}(T)=-4 \sum_{i=0, \pm} \int_{k, n} \log \left[\left(\omega_{n}^{i}\right)^{2}+\vec{k}^{2}+M^{2}\left(\left(\omega_{n}^{i}\right)^{2}\right)\right]+\frac{m_{d}^{2}}{2 G}+\mathcal{U}(\Phi, \bar{\Phi})
$$

where the notation $\int_{k, n}=T \sum_{n} \int d^{3} k /(2 \pi)^{3}$ has been introduced. $\Phi$ denotes the Polyakov loop variable, which is given by $\Phi=\frac{1}{3} \operatorname{Tr}_{c} e^{i \phi / T}$. Here $\phi \equiv A_{4}=i A_{0}$ is related to the (Euclidean) background gauge field. In Polyakov gauge it is diagonal in color space, i.e., $\phi=\phi_{3} \lambda_{3}+\phi_{8} \lambda_{8}$. Following Ref. [8], we require $\Phi=\bar{\Phi}$ to be real with real $\phi_{3}, \phi_{8}$. As a consequence $\phi_{8}=0$ and we are left with one variable $\phi_{3}$ [8].

Due to the coupling to the Polyakov loop the fermionic Matsubara frequencies $\omega_{n}=$ $(2 n+1) \pi T$ are shifted:

$$
\omega_{n}^{ \pm}=\omega_{n} \pm \phi_{3}, \quad \omega_{n}^{0}=\omega_{n}
$$

For the Polyakov loop potential $\mathcal{U}(\Phi, \bar{\Phi})$ we adopt the the logarithmic form of Ref. [8], which has been fitted to the quenched lattice data of Ref. [24].

The order parameters (mean field values for $m_{d}$ and $\phi_{3}$ ) are obtained by minimization of the mean-field part of the thermodynamic potential $\frac{\partial \Omega_{\mathrm{mf}}}{\partial m_{d}}=0, \frac{\partial \Omega_{\mathrm{mf}}}{\partial \phi_{3}}=0$.

In Fig. 1 we show the resulting temperature dependence of the quark condensate $\langle\bar{q} q\rangle^{T}$ (normalized to its vacuum value) and of the Polyakov loop expectation value. We also show the corresponding results for the case of uncoupled (pure) quark and gluon sectors. In the pure quark model the critical temperature for chiral restoration is $T_{c}=116 \mathrm{MeV}$, whereas the pure gauge sector has a critical temperature for deconfinement $T_{d}=270 \mathrm{MeV}$, fixed 
from lattice data for the Polyakov loop. When the quark and gluon sectors are coupled these temperatures get synchronized so that $T_{c} \approx T_{d} \approx 200 \mathrm{MeV}$.

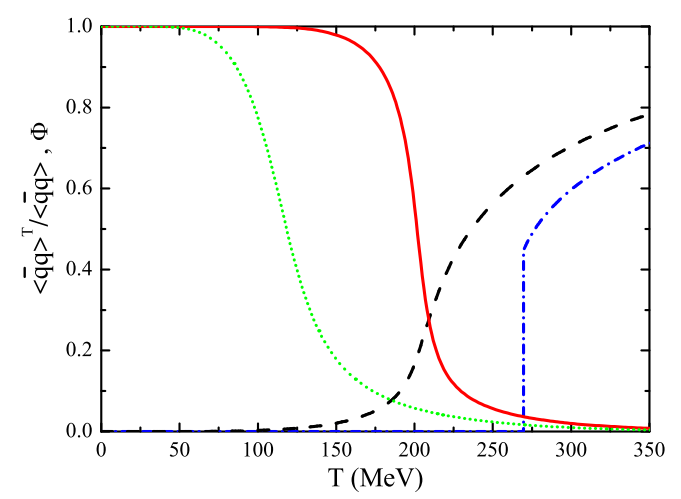

Figure 1. Quark condensate (normalized to its vacuum value) in the nonlocal NJL model (green dotted line) and the nonlocal PNJL model (red solid line) compared to the Polyakov loop in pure gauge theory (blue dash-dotted line) and in the nonlocal PNJL model (black dashed line).

We now include $\Omega_{\text {corr }}$ to study the effect of mesonic correlations. The properties of mesons in the PNJL model have been studied in Ref. [7] and the generalization to our nonlocal model is straightforward. Key ingredients are the quark-antiquark polarization loops $\Pi_{M}$. Using the notations $D\left(k^{2}\right)=k^{2}+M^{2}\left(k^{2}\right), k_{n+}^{i}=\left(\omega_{n}^{i}+\nu_{m}, \vec{k}+\vec{p}\right)$, and $k_{n}^{i}=\left(\omega_{n}^{i}, \vec{k}\right)$ they can be written as

$$
\left.\Pi_{\pi, \sigma}\left(\vec{p}, \nu_{m}\right)=4 N_{f} \sum_{i=0, \pm} \int_{k, n} \frac{f^{2}\left(\left(k_{n+}^{i}\right)^{2}\right) f^{2}\left(\left(k_{n}^{i}\right)^{2}\right)}{D\left(\left(k_{n+}^{i}\right)^{2}\right) D\left(\left(k_{n}^{i}\right)^{2}\right)}\left[k_{n+}^{i} \cdot k_{n}^{i} \pm M\left(\left(k_{n+}^{i}\right)^{2}\right) M\left(\left(k_{n}^{i}\right)^{2}\right)\right)\right] .
$$

The mesonic contributions to the thermodynamic potential are then (to lowest order) given by the ring sum [17],

$$
\Omega_{\mathrm{corr}}(T)=\sum_{M=\pi, \sigma} \frac{d_{M}}{2} \int_{p, m} \ln \left[1-G \Pi_{M}\left(\vec{p}, \nu_{m}\right)\right]
$$

with the mesonic degeneracy factor $d_{M}$.

The fermionic Matsubara sum, the three-momentum integration and the sum over the three color modes $i=0,+,-$ in $(7)$ are evaluated numerically with a finite result for $\Pi_{\pi}\left(\vec{p}, \nu_{m}\right)$ to be inserted in Eq. (8). The bosonic Matsubara sum in (8) and the three momentum integral are also performed numerically.

Our model predictions for the the pressure $P(T)=-\Omega(T)$, divided by the StefanBoltzmann limit, are displayed in Fig. 2. For comparison with the full result we also show 
the mean-field result and the mean-field plus pion contribution as well as the result for an ideal pion and sigma gas with the masses fixed at their vacuum values. We find that at low temperatures the mean-field (i.e., quark) contribution is suppressed and the pressure can be well described by a free pion gas. Near the critical temperature the $\sigma$ meson gives an additional visible contribution whereas already after $T>1.5 T_{c}$ the mesonic contributions are negligible and the quark-gluon mean-field dominates the pressure.

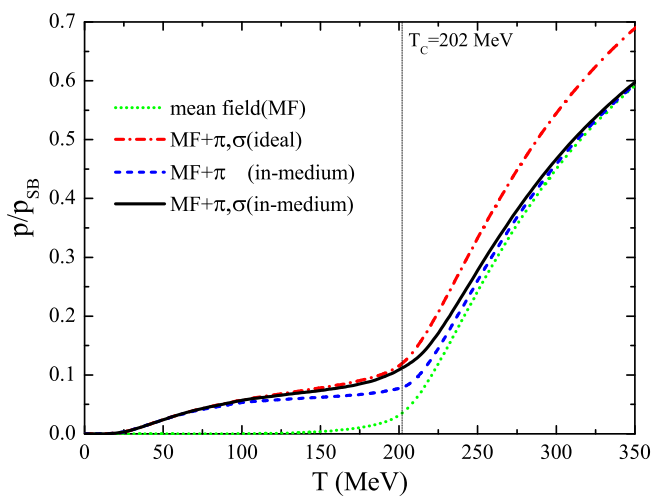

Figure 2. Scaled pressure $p / p_{S B}$ in the nonlocal PNJL model with the physical pion mass: mean field contribution (green dotted line), mean field + pion (blue dashed line), mean field + pion + sigma (black solid line). The red dash-dotted line denotes the scaled pressure of an ideal pion + sigma gas with fixed masses.

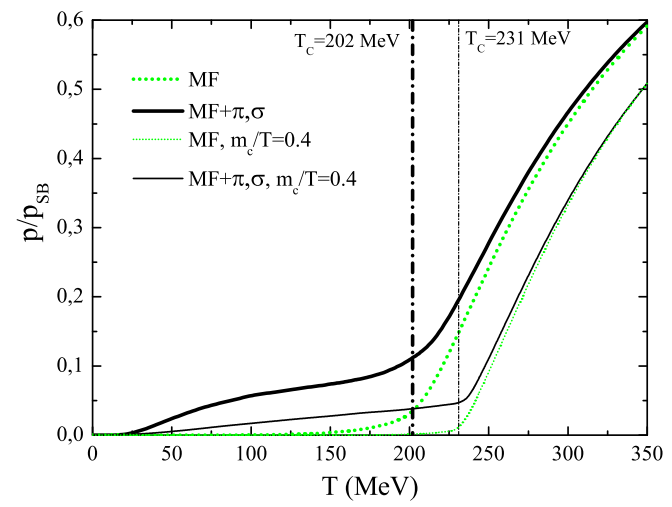

Figure 3. Scaled pressure $p / p_{S B}$ in the nonlocal PNJL model with the physical pion mass (thick lines) and with $m_{c} / T=0.4$ (thin lines): mean field contribution (green dotted line), mean field + pion + sigma (black solid line).

So far we have used the parameter set of Ref. [22] with $m_{c}=5.8 \mathrm{MeV}$, corresponding to the physical pion mass of $140 \mathrm{MeV}$ in vacuum. On the other hand, in most lattice calculations 
the masses are considerably larger. Thus in order to perform a meaningful comparison with lattice results, we should repeat our model calculations using quark masses similar to the lattice ones. To be specific, we choose the current quark mass to scale with the temperature as $m_{c}=0.4 T$, mimicking the situation in the lattice calculation of Ref. [25], where the up and down quark masses behave in the same way.

Our results for the scaled pressure are displayed in Fig. 3. For comparison we show again the results obtained with $m_{c}=5.8 \mathrm{MeV}$ (thick lines). With $m_{c}=0.4 T$ (thin lines) the qualitative behavior remains unchanged: Above $T_{c}$ the total pressure (black solid line) converges quickly to the mean-field result (green dotted line), whereas below $T_{c}$ the pressure is dominated by the mesonic contribution. Quantitatively, the meson contributions are of course strongly suppressed in the case of the heavier quark masses. Also note that the meanfield result is affected as well. In particular $T_{c}$ rises from $202 \mathrm{MeV}$ for $m_{c}=5.8 \mathrm{MeV}$ to $231 \mathrm{MeV}$ for $m_{c}=0.4 T$

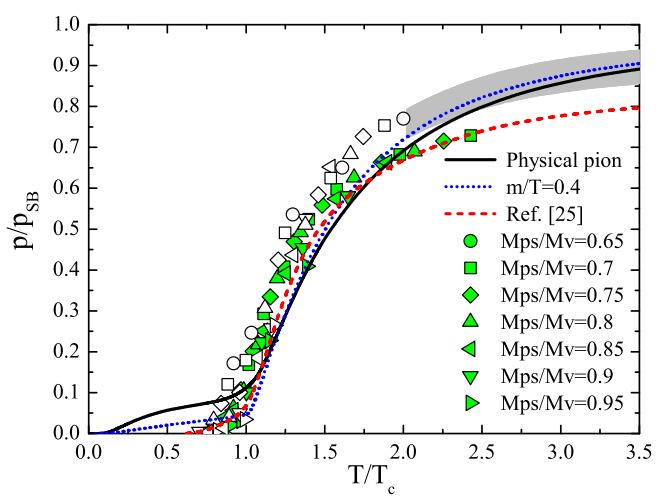

Figure 4. Scaled pressure $p / p_{S B}$ as a function of $T / T_{c}$ : non-local PNJL model with physical pion mass (black solid line) and with $m_{c} / T=0.4$ (blue dotted line). Red dashed line: Lattice data for two-flavor QCD with staggered quarks [25]. The shaded region is an estimated continuum extrapolation of these data for massless QCD [25]. Points: Lattice data for two-flavor QCD with Wilson-type quarks [11] for $N_{t}=6$ (open symbols) and $N_{t}=4$ (green filled symbols). The data for the pressure [11] have been divided by the Stefan-Boltzmann limit for $N_{t}=6$ and $N_{t}=4$, respectively, as given in Ref. [11].

In Fig. 4 we compare our model results for the scaled pressure with lattice simulations. Our model results are indicated by the black solid line and the blue dotted line, which correspond to the physical parameterization $\left(m_{c}=5.8 \mathrm{MeV}\right)$ and the case $m_{c}=0.4 T$, respectively. Both curves include mesonic correlations and are identical to the black solid 
lines in Fig. 3. Note, however, that they are now displayed as functions of $T / T_{c}$, with $T_{c}$ being different for the two parameterizations.

The red dashed line indicates the result of Ref. [25] obtained on a $16^{3} \times 4$ lattice with improved staggered fermion actions for two flavors. As mentioned above, in this calculation the quark mass is unphysically large and scales with the temperature, $m_{u, d} / T=0.4$. At $T \gtrsim 2 T_{c}$ our results are systematically above the lattice data. Since the latter are obtained with four time slices only $\left(N_{t}=4\right)$, this is probably a lattice artifact. This interpretation is supported by the lattice results of Ref. [11] for Wilson fermions with $N_{t}=4$ (green filled symbols) and $N_{t}=6$ (open symbols). While the former almost coincide with the results of Ref. [25], the latter indicate higher pressures at large T. Although the Wilson data have been extracted on "lines of constant physics", i.e., for $T$-independent quark masses and are therefore not entirely comparable to the staggered fermion data, this tendency should be correct. Indeed, the authors of Ref. [25] tried a continuum extrapolation for massless QCD (shaded area), which is in fair agreement with our model results for both, physical and large quark masses.

Whereas in our model, even for $m_{c}=0.4 T$, there remains a visible pion contribution to the pressure down to $T / T_{c} \approx 0.2$, the lattice pressure of [25] vanishes at $T / T_{c}=0.6$. This discrepancy has a trivial explanation by the fact that the lattice pressure has been obtained by the "integral method" which leaves one integration constant undetermined. In Ref. [25], this constant has been fixed by the choice that the pressure vanishes at $T / T_{c}=0.6$. On the other hand, chiral perturbation theory predicts that at very low temperature the pressure is well described by an ideal pion gas, in good agreement with our model. Hence, if the integration constant on the lattice had been fitted to $\chi P T$, rather than setting the pressure at $T=0.6 T_{c}$ equal to zero, they would be in good agreement with our results at this point. On the other hand, our approach underestimates the lattice data in the region $0.9 \lesssim T / T_{c} \lesssim 1.6$. This may be attributed to our neglect of hadronic resonances, other than pion and sigma.

In summary, we have extended previous studies of the thermodynamics of low-energy QCD within Polyakov-loop NJL models to include mesonic correlations. To that end, we have evaluated the pion and sigma contributions to the pressure by calculating the ring sum. We have thereby employed a nonlocal four-fermion interaction of the instanton liquid type. The pionic contribution dominates the pressure in the low temperature region. In 
this regime, the pressure is quite insensitive to the details of the interaction and agrees

almost exactly with that of an ideal pion gas. At temperatures $\gtrsim T_{c}$, on the other hand, the mesonic contributions die out. For unphysical pion masses, as obtained in most present lattice calculations, mesonic correlations play only a minor role, even below $T_{c}$, in accordance with the lattice results.

For the future we plan to include the back reaction effect of mesonic correlations to the equation of motion of mean fields. This can lead to a lowering of $T_{c}$. We also plan to study nonzero chemical potentials, which may require a nontrivial extrapolation of the Polyakovloop potential into this regime [26]. We then should include baryonic degrees of freedom as well.

\section{ACKNOWLEDGMENTS}

We thank O. Kaczmarek, C. Sasaki, and K. Redlich for critial remarks and illuminating discussions. A.E.R. thanks J. Wambach for his hospitality at TU Darmstadt. We acknowledge support by the Heisenberg-Landau programme (M.B., A.E.R., M.K.V), by the Russian Foundation for Basic Research under contract 05-02-16699 (A.E.R., M.K.V), by BMBF (A.E.R) and by the Polish Ministry of Science and Higher Education (D.B.).

1. B. Müller and J. L. Nagle, Ann. Rev. Nucl. Part. Sci. 56, 93 (2006).

2. U.-G. Meißner and G. Schierholz, arXiv:hep-ph/0611072.

3. M. S. Bhagwat, M. A. Pichowsky, C. D. Roberts and P. C. Tandy, Phys. Rev. C 68, 015203 (2003); C. S. Fischer and M. R. Pennington, Phys. Rev. D 73, 034029 (2006).

4. A. Bender, D. Blaschke, Y. Kalinovsky and C. D. Roberts, Phys. Rev. Lett. 77, 3724 (1996)

5. C. Ratti, M. A. Thaler and W. Weise, Phys. Rev. D 73, 014019 (2006).

6. P. N. Meisinger and M. C. Ogilvie, Phys. Lett. B 379, 163 (1996); K. Fukushima, Phys. Lett. B 591, 277 (2004); E. Megías, E. Ruiz Arriola and L. L. Salcedo, Phys. Rev. D 74, 065005 (2006); C. Sasaki, B. Friman and K. Redlich, Phys. Rev. D 75, 074013 (2007).

7. H. Hansen, W. M. Alberico, A. Beraudo, A. Molinari, M. Nardi and C. Ratti, Phys. Rev. D 75, 065004 (2007).

8. S. Rößner, C. Ratti and W. Weise, Phys. Rev. D 75, 034007 (2007). 
9. Y. Nambu and G. Jona-Lasinio, Phys. Rev. 122, 345 (1961); 124, 246 (1961).

10. C. R. Allton, S. Ejiri, S. J. Hands, O. Kaczmarek, F. Karsch, E. Laermann and C. Schmidt, Phys. Rev. D 68, 014507 (2003).

11. A. Ali Khan et al. [CP-PACS collaboration], Phys. Rev. D 64, 074510 (2001).

12. G. 't Hooft, Nucl. Phys. B 72, 461 (1974).

13. E. Quack and S. P. Klevansky, Phys. Rev. C 49 (1994) 3283; D. Ebert, M. Nagy and M. K. Volkov, Phys. Atom. Nucl. 59, 140 (1996); V. Dmitrasinovic, H. J. Schulze, R. Tegen and R. H. Lemmer, Annals Phys. 238 (1995) 332; E. N. Nikolov, W. Broniowski, C. V. Christov, G. Ripka and K. Goeke, Nucl. Phys. A 608, 411 (1996); D. Blaschke, Y. L. Kalinovsky, G. Röpke, S. M. Schmidt and M. K. Volkov, Phys. Rev. C 53, 2394 (1996); M. Oertel, M. Buballa and J. Wambach, Phys. Atom. Nucl. 64, 698 (2001).

14. E. V. Shuryak and I. Zahed, Phys. Rev. D 70, 054507 (2004).

15. N. F. Mott, Rev. Mod. Phys. 40, 677 (1968).

16. H. Satz, Nucl. Phys. A 418 (1984) 447c; D. Blaschke, F. Reinholz, G. Röpke and D. Kremp, Phys. Lett. B 151 (1985) 439.

17. J. Hüfner, S. P. Klevansky, P. Zhuang and H. Voss, Annals Phys. 234 (1994) 225; P. Zhuang, J. Hüfner and S. P. Klevansky, Nucl. Phys. A 576 (1994) 525.

18. M. Schmidt, G. Röpke and H. Schulz, Ann. Phys. 202, 57 (1990).

19. G. Ripka, "Quarks bound by chiral fields: The quark-structure of the vacuum and of light mesons and baryons," Oxford, UK: Clarendon Pr. (1997).

20. E. V. Shuryak, Nucl. Phys. B 203, 93 (1982); D. Diakonov and V. Y. Petrov, Nucl. Phys. B 245, 259 (1984); Nucl. Phys. B 272, 457 (1986).

21. A. E. Dorokhov and L. Tomio, Phys. Rev. D 62, 014016 (2000).

22. D. Gomez Dumm, A. G. Grunfeld and N. N. Scoccola, Phys. Rev. D 74, 054026 (2006).

23. R. D. Bowler and M. C. Birse, Nucl. Phys. A 582, 655 (1995).

24. O. Kaczmarek, F. Karsch, P. Petreczky and F. Zantow, Phys. Lett. B 543, 41 (2002).

25. F. Karsch, E. Laermann and A. Peikert, Phys. Lett. B 478, 447 (2000).

26. B.-J. Schaefer, J. M. Pawlowski and J. Wambach, Phys. Rev. D 76, 0704023 (2007). 


\title{
ЭФФЕКТ МЕЗОННЫХ КОРЕЛЛЯЦИЙ В ФАЗОВОМ ПЕРЕХОДЕ КХД
}

\author{
Д. Блашке, М. Бубала, А.Е. Раджабов, М.К. Волков \\ Фазовый переход сильновзаимодействующей материи при конечной температуре \\ изучен в нелокальной киральной кварковой модели типа НИЛ соединенной с пет- \\ лей Полякова. В отличие от существующих исследований в рамках среднего поля \\ в модель включены мезонные корреляции с помощью кварк-антикварковой коль- \\ цевой суммы. Для физических масс пиона нами обнаружено, что пионы дают до- \\ минирующий вклад в давление, тогда как выше $T_{c}$ давление хорошо описывается \\ результатом вычислений в приближении среднего поля. Для большой массы пиона, \\ как в вычислениях на решетке, мезонные эффекты подавлены.
}

